\title{
Pregnancy loss and psychiatric disorders in young women: an Australian birth cohort study
}

\author{
Kaeleen Dingle, Rosa Alati, Alexandra Clavarino, Jake M. Najman and Gail M. Williams
}

\section{Background}

Recent evidence has linked induced abortion with later adverse psychiatric outcomes in young women.

\begin{abstract}
Aims
To examine whether abortion or miscarriage are associated with subsequent psychiatric and substance use disorders.
\end{abstract}

\section{Method}

A sample ( $n=1223)$ of women from a cohort born between 1981 and 1984 in Australia were assessed at 21 years for psychiatric and substance use disorders and lifetime pregnancy histories.

\section{Results}

Young women reporting a pregnancy loss had nearly three times the odds of experiencing a lifetime illicit drug disorder (excluding cannabis): abortion odds ratio (OR) $=3.6$ (95\% Cl 2.0-6.7) and miscarriage OR=2.6 (95\% Cl 1.2-5.4). Abortion was associated with alcohol use disorder (OR=2.1, $95 \% \mathrm{Cl} 1.3-3.5)$ and 12-month depression $(\mathrm{OR}=1.9,95 \% \mathrm{Cl}$ 1.1-3.1).

\section{Conclusions}

These findings add to the growing body of evidence suggesting that pregnancy loss per se, whether abortion or miscarriage, increases the risk of a range of substance use disorders and affective disorders in young women.

\section{Declaration of interest}

None. Funding detailed in Acknowledgements.
Recent evidence suggests induced abortion may be associated with later psychiatric disorders in young women. ${ }^{1-3}$ Such findings may question one of the main orthodoxies underpinning legalised abortion, that of preventing adverse psychiatric outcomes after an unwanted pregnancy. ${ }^{2}$ It has been observed that the associations can be explained by previous depression or drug misuse, or that the rates of psychiatric morbidity reported after abortion are no different than expected in young women of childbearing age. ${ }^{4-7}$ Alternatively, psychiatric disorders after abortion may be a result of the experience of pregnancy loss in general, rather than being specific to induced abortion. Miscarriage and pregnancy are associated with significant psychiatric morbidity. Anxiety, depression and substance use disorders are associated with miscarriage, ${ }^{8}$ and the risk of severe psychosis increases for some women during pregnancy. ${ }^{9}$ To date, no population study has directly compared psychiatric outcomes in women after abortion and miscarriage. This study used prospective data to examine the associations between pregnancy loss, whether from induced abortion or spontaneous miscarriage, and a range of DSM-IV ${ }^{10}$ psychiatric disorders assessed at age 21 years.

\section{Methods}

\section{Participants}

Data was taken from the Mater-University of Queensland study of pregnancy and its outcomes (MUSP), ${ }^{11}$ a 21 -year prospective birth cohort study that investigates the health and behaviour of mother-child pairs. The baseline female cohort $(n=3474)$ were born between 1981 and 1984 at a major metropolitan public hospital in Brisbane, Australia. Mother-daughter pairs were subsequently reassessed at 6 months, 5, 14, and 21 years after birth. During the 21-year follow-up, over the years 2000-2004, all the original mother-child pairs were recontacted; however, resource constraints limited our ability to interview people residing outside of the greater Brisbane area and the 7-year gap between follow-ups made it difficult to regain contact with some. There were few who refused to participate. No social or other characteristics were used to screen inclusion, although there was a modest over-representation of disadvantage in women lost to follow-up (online Table DS1). At 21-year follow-up, 1988 female children (57.2\% of the baseline cohort), now young women, were interviewed. Of these, $61.5 \%(n=1223)$ had complete data on lifetime pregnancy outcomes, psychiatric diagnoses at age 21 and covariates from birth, 14 and 21 years. Ethical approval for each phase was obtained from the University of Queensland.

\section{Measurement of psychiatric disorders}

At 21-year follow-up, the lifetime version of the Composite International Diagnostic Interview computerised version (CIDIAuto, version 2.1) $)^{12}$ was used to assess psychiatric disorders. The CIDI has been found to have good reliability and validity. ${ }^{13}$ The study reports six DSM-IV ${ }^{10}$ disorders: total affective and anxiety disorders; alcohol, cannabis and other illicit drug use and dependence disorders, as well as nicotine withdrawal and dependence disorders. The age at first onset of disorder and disorders occurring within the previous 12 months were used. Although we had intended to explore associations between pregnancy outcomes and specific psychiatric disorders such as psychosis, only 21 women met DSM-IV criteria for any psychosis. We were, therefore, unable to undertake meaningful analysis of the influence of pregnancy outcomes on psychotic disorders.

\section{Measurement of pregnancy outcomes}

Women reported the number of previous pregnancies, abortions, miscarriages and births they had experienced by age 21 . We created a variable which incorporated all four possibilities (never pregnant, live birth only, ever had an abortion and ever miscarried) (Fig. 1). Seventeen women had both an abortion and miscarriage: for the analysis, six women who had miscarried two or three times were coded to miscarriage, five women who had had two abortions were coded to abortion, and the remaining six women with one abortion and one miscarriage were randomly 


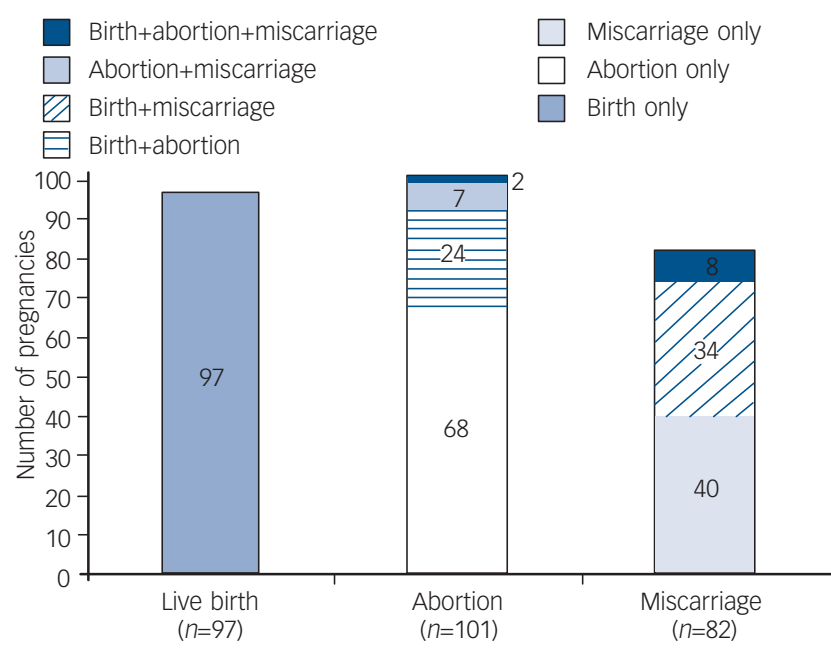

Fig. 1 Pregnancy outcomes for 280 women reporting a pregnancy at 21 years $(n=1223$, excludes 943 never pregnant women, includes 17 women who had both an abortion and miscarriage).

assigned to either the abortion or miscarriage group. Those with missing data in any of the questions were excluded.

\section{Confounders}

Confounders included maternal and familial factors, measures related to pre-existing behaviour problems and substance misuse, as well as demographic characteristics of the young women.

\section{Young women's early life and family background}

Symptoms of anxiety and depression experienced by the young women's mothers during pregnancy were assessed using the two seven-item sub-scales of the Delusions-Symptoms-States Inventory (DSSI). ${ }^{14}$ The depression sub-scale strongly correlates with DSM-IV major depressive disorder. ${ }^{15}$ Mothers were classified as anxious or depressed if they reported four or more symptoms. Maternal smoking was assessed pre-pregnancy and at birth. At 14-year follow-up, mothers indicated if they lived with the young women's biological father, lived alone or with others. Mother-child communication was measured with a problem communication sub-scale from the Parent-Adolescent Communication Scale (PACS). ${ }^{16}$ Family socio-economic status was assessed by yearly family income and maternal education at baseline.

\section{Adolescent behaviour}

At age 14, we used maternal reports of the young women's delinquent/aggressive behaviour from the Achenbach's Child Behavior Checklist (CBCL) and the Youth Self-Report (YSR) anxious/ depressed sub-scale. ${ }^{17}$ The checklists are widely used, standardised scales that are reliable, valid indicators of problem behaviour. ${ }^{18}$ At 14 , the young women were asked to define their overall school performance (average or below and above average).

At age 21, participants were asked whether they used cannabis and the age this was initiated. Participants were also asked the age at which they first had sexual intercourse, women reporting no sexual intercourse were excluded. A history of exposure to sexual violence or child sexual abuse and the age this first occurred was obtained with items from the Los Angeles epidemiologic catchment area project. ${ }^{19}$ Child sexual abuse was defined as unwanted sexual contact before 16 years of age with a person 5 or more years their senior. Young women were also asked if they had ever been raped.

\section{Demographic factors at age 21}

Demographic factors included age, marital status, age of leaving the family home, living arrangements (parents/relatives, renter, boarder or owner) and education level at age 21.

\section{Statistical analysis}

Chi-squared tests and logistic regression were used to explore the bivariate associations of pregnancy outcomes and each DSM-IV disorder. We then fitted multivariate logistic models on a restricted sample of young women $(n=1223)$ with complete data for each DSM-IV disorder separately. Associations with each disorder were adjusted consecutively for maternal and family factors at baseline and 14-year follow-up (model 1), adolescent behaviour problems at 14 (model 2), adolescent behaviour reported at 21 (model 3 ) and concurrent demographic factors (model 4$)$. Clearly non-significant factors $(P>0.1$ or those with unstable estimates as assessed by large standard errors) were removed. Each model was reassessed until only significant effects $(P<0.05)$ remained. This was undertaken separately for each of the six DSM-IV disorders. Unadjusted and adjusted odds ratios (OR) and 95\% confidence intervals were used to estimate the association between pregnancy outcomes and each disorder compared with the never pregnant group. All pairwise pregnancy outcome comparisons were undertaken with each of the fully adjusted models.

\section{Sensitivity analyses}

A series of sensitivity analyses were done to further explore the timing of the associations and effects of substance misuse, behaviour problems and psychiatric morbidity that possibly preceded pregnancy loss. Factors such as adolescent cigarette smoking, alcohol consumption and anxious/depressed and aggressive/ delinquent behaviour were excluded from the model as these are possible causal factors associated with later psychiatric morbidity. Six separate sensitivity analyses were conducted:

(a) current (12-month) diagnoses were used instead of lifetime diagnoses;

and exclusion of women who reported:

(b) both a miscarriage and abortion;

(c) giving birth and pregnancy loss either as an abortion or miscarriage or both;

(d) smoking, drinking alcohol or anxious/depressed problem behaviours at 14 years;

(e) aggressive/delinquent behaviours at 14 years; and

(f) onset of a particular DSM-IV disorder at or before the age of sexual debut.

Finally, we used inverse probability weighting ${ }^{20}$ to determine whether loss to follow-up affected the validity of our findings. This was done for the six DSM-IV diagnoses both lifetime and current (12-month) disorders. Analyses were carried out using SPSS version 15.0 and STATA version 9.0 on Windows.

\section{Results}

Overall, 1223 young women had complete data. By about 20 years (s.d.=0.83) 943 reported never being pregnant and $280(22.9 \%)$ reported one or more pregnancies. Over a quarter of those 
pregnant $(26.8 \%, n=75 ; 6.1 \%$ of the sample) had complex lifetime pregnancy histories with two or three outcomes (Fig. 1).

\section{Associations between pregnancy and psychiatric disorders}

The unadjusted odds of having a lifetime psychiatric or substance use disorder were highest among women reporting a pregnancy loss and lowest among women who never had a pregnancy or gave birth; women with a nicotine disorder were the exception. Women with a history of abortion or miscarriage were twice as likely to meet criteria for alcohol, cannabis, other illicit drug use, depressive or anxiety disorder compared with those who were never pregnant or who had given birth (Table 1). Young women who became pregnant regardless of outcome had similarly high odds of having a lifetime tobacco dependency compared with never pregnant women.

\section{Adjustment for confounding}

Online Table DS2 shows associations between pregnancy outcomes and a number of potential confounding factors. Pregnancy outcomes were associated with greater social and economic disadvantage, family instability, alcohol and cigarette use, aggressive/delinquent behaviours, early sexual intercourse and poorer educational outcomes at both age 14 and 21 . There were only modest differences between women reporting an abortion and those with a history of miscarriage or birth.

Table 2 shows the multivariable logistic regression analyses. Women reporting a pregnancy were twice as likely to develop a tobacco dependency, with the association being slightly attenuated by adjustment for maternal and family factors and adolescent behaviour. Women who reported an abortion had twice the odds of having an alcohol disorder and nearly four times the odds of an illicit drug use disorder (excluding cannabis). Also, those reporting an abortion had an increased risk of affective disorder, although this association bordered significance after adjustment for 21-year demographic factors. Women who reported a miscarriage had a similarly high risk of developing an illicit drug use disorder, but not affective or alcohol disorders, as women reporting an abortion.

\section{Comparison testing of pregnancy outcomes}

For all psychiatric outcomes, except for tobacco dependence, pairwise comparisons show that risk did not vary significantly between the never pregnant and live birth groups $(P>0.15)$ and importantly, between women reporting either a miscarriage or abortion $(P>0.40)$. Young women reporting a pregnancy regardless of outcome were at least twice as likely to have a tobacco dependency disorder as never pregnant women $(P<0.02)$ and the risk of disorder did not vary between pregnancy outcomes $(P>0.64)$. Women reporting a pregnancy loss were at increased risk of affective disorders, irrespective of whether the loss was due to abortion $(P=0.02)$ or miscarriage $(P=0.05)$. Those reporting having an abortion were also more likely to develop an alcohol disorder $(P=0.02)$ or an illicit drug use disorder $(P=0.02)$, although not a cannabis disorder, than women giving birth (online Table DS3).

\section{Sensitivity analyses}

In this birth cohort study, DSM-IV diagnoses and pregnancy outcomes were concurrently measured, making the direction of the associations difficult to determine. Therefore a series of six sensitivity analyses were undertaken. When we excluded women with complex pregnancy outcomes (17 participants reporting both an abortion and miscarriage and 68 reporting one or more types of pregnancy loss as well as a birth) there was no substantial change to our results, with the exception of the association between abortion and anxiety disorder, which became significant $(\mathrm{OR}=1.6,95 \% \mathrm{CI} 1.1-2.7)$ when individuals with abortion and miscarriage were removed, although the same was not seen when women reporting at least one birth and pregnancy loss were

\begin{tabular}{|c|c|c|c|c|c|}
\hline Lifetime DSM-IV disorders & $\begin{array}{l}\text { Never pregnant }{ }^{\mathrm{a}} \\
\quad(n=943)\end{array}$ & $\begin{array}{l}\text { Live birth } \\
\qquad(n=97)\end{array}$ & $\begin{array}{l}\text { Abortion } \\
(n=101)\end{array}$ & $\begin{array}{l}\text { Miscarriage } \\
\quad(n=82)\end{array}$ & $P^{\mathrm{b}}$ \\
\hline \multicolumn{6}{|c|}{ Nicotine (withdrawal and dependence) $(n=198)$} \\
\hline Prevalence, \% & 11.8 & 26.0 & 34.7 & 32.9 & \multirow[t]{2}{*}{$<0.001$} \\
\hline $\mathrm{OR}(95 \% \mathrm{Cl})^{\mathrm{C}}$ & 1.0 & $2.6(1.6-4.3)$ & $4.0(2.5-6.3)$ & $3.7(2.2-6.1)$ & \\
\hline \multicolumn{6}{|c|}{ Alcohol (misuse and dependence) $(n=214)$} \\
\hline Prevalence, \% & 14.7 & 16.8 & 35.6 & 29.6 & \multirow[t]{2}{*}{$<0.001$} \\
\hline $\mathrm{OR}(95 \% \mathrm{Cl})$ & 1.0 & $1.2(0.7-2.1)$ & $3.2(2.1-5.0)$ & $2.4(1.5-4.1)$ & \\
\hline \multicolumn{6}{|c|}{ Cannabis (misuse and dependence) $(n=172)$} \\
\hline Prevalence, \% & 11.0 & 15.5 & 31.7 & 25.6 & \multirow[t]{2}{*}{$<0.001$} \\
\hline OR $(95 \% \mathrm{Cl})$ & 1.0 & $1.5(0.8-2.7)$ & $3.7(2.3-6.0)$ & $2.8(1.6-4.7)$ & \\
\hline \multicolumn{6}{|c|}{$\begin{array}{l}\text { Other illicit substance use } \\
\text { (misuse and dependence) (n=93) }\end{array}$} \\
\hline Prevalence, \% & 5.4 & 6.3 & 21.8 & 17.3 & \multirow[t]{2}{*}{$<0.001$} \\
\hline OR $(95 \% \mathrm{Cl})$ & 1.0 & $1.2(0.5-2.8)$ & $4.8(2.8-8.4)$ & $3.6(1.9-7.0)$ & \\
\hline \multicolumn{6}{|l|}{ Affective $^{\mathrm{e}}(n=344)$} \\
\hline Prevalence, \% & 25.1 & 28.7 & 45.5 & 43.2 & \multirow[t]{2}{*}{$<0.001$} \\
\hline OR $(95 \% \mathrm{Cl})$ & 1.0 & $1.2(0.8-1.9)$ & $2.5(1.6-3.8)$ & $2.3(1.4-3.6)$ & \\
\hline \multicolumn{6}{|l|}{ Anxiety $^{f}(n=418)$} \\
\hline Prevalence, \% & 30.1 & 41.1 & 51.5 & 53.7 & \multirow[t]{2}{*}{$<0.001$} \\
\hline OR $(95 \% \mathrm{Cl})$ & 1.0 & $1.6(1.1-2.5)$ & $2.5(1.6-3.7)$ & $2.7(1.7-4.2)$ & \\
\hline \multicolumn{6}{|c|}{$\begin{array}{l}\text { a. Reference group. } \\
\text { b. } \chi^{2} \text {-tedt of independence. } \\
\text { c. Unadjusted OR with } 95 \% \mathrm{Cl} \text { obtained using binominal logistic regression. } \\
\text { d. Other illicit substance use excluding cannabis, includes reported use of one or more of the following substances: opioids, sedatives, cocaine, amphetamines, hallucinogens, } \\
\text { inhalants, phencyclidine and other undetermined substances. } \\
\text { e. Includes any affective disorder: major depressive disorder (mild, moderate or severe), dysthymia and bipolar disorders. } \\
\text { f. Includes any anxiety disorder: generalised anxiety disorder, post-traumatic stress disorder, any phobia and/or any panic disorder. }\end{array}$} \\
\hline
\end{tabular}




\begin{tabular}{|c|c|c|c|c|c|c|}
\hline \multirow[b]{2}{*}{ Model } & \multicolumn{6}{|c|}{ DSM-IV disorders at 21-year follow-up, OR (95\% CI) } \\
\hline & Nicotine & Alcohol & Cannabis & $\begin{array}{c}\text { Other illicit } \\
\text { substance use }\end{array}$ & Affective & Anxiety \\
\hline \multicolumn{7}{|c|}{ 1. Adjusted for early life and family factors $s^{a, b}$} \\
\hline Live birth & $2.7(1.6-4.5)$ & $1.1(0.6-1.9)$ & $1.3(0.7-2.3)$ & $1.4(0.6-3.3)$ & $1.0(0.6-1.7)$ & $1.4(0.9-2.1)$ \\
\hline Abortion & $4.0(2.5-6.4)$ & $2.8(1.8-4.4)$ & $3.3(2.1-5.4)$ & $5.8(3.3-10.4)$ & $2.2(1.4-3.5)$ & $2.0(1.3-3.1)$ \\
\hline Miscarriage & $3.6(2.1-6.2)$ & $2.2(1.3-3.7)$ & $2.5(1.5-4.4)$ & $4.1(2.1-8.2)$ & $2.1(1.3-3.5)$ & $2.3(1.4-3.6)$ \\
\hline \multicolumn{7}{|c|}{$\begin{array}{l}\text { 2. Adjusted for previous significant factors } \\
\text { and adolescent behaviour }{ }^{a, c}\end{array}$} \\
\hline Live birth & $2.4(1.4-4.1)$ & $0.8(0.5-1.5)$ & $1.0(0.6-2.0)$ & $1.1(0.4-2.8)$ & $0.9(0.5-1.5)$ & $1.2(0.8-1.9)$ \\
\hline Abortion & $3.3(2.0-5.4)$ & $2.3(1.4-3.8)$ & $2.6(1.6-4.3)$ & $4.4(2.4-8.0)$ & $1.9(1.2-3.0)$ & $1.8(1.2-2.8)$ \\
\hline Miscarriage & $3.1(1.8-5.4)$ & $1.9(1.1-3.2)$ & $2.1(1.2-3.8)$ & $4.0(2.0-8.1)$ & $2.0(1.2-3.4)$ & $2.1(1.3-3.4)$ \\
\hline \multicolumn{7}{|c|}{$\begin{array}{l}\text { 3. Adjusted for previous significant factors } \\
\text { and adolescent factors reported at } 21^{a, c, d}\end{array}$} \\
\hline Live birth & $2.4(1.4-4.2)$ & $0.8(0.4-1.5)$ & $0.8(0.4-1.4)$ & $1.0(0.4-2.6)$ & $0.8(0.5-1.4)$ & $1.1(0.7-1.8)$ \\
\hline Abortion & $2.6(1.6-4.3)$ & $2.0(1.2-3.3)$ & $1.7(1.0-2.8)$ & $3.6(2.0-6.7)$ & $1.7(1.1-2.7)$ & $1.5(1.0-2.5)$ \\
\hline Miscarriage & $2.3(1.3-4.3)$ & $1.5(0.8-2.7)$ & $1.5(0.8-2.6)$ & $2.6(1.2-5.4)$ & $1.7(1.0-3.0)$ & $1.7(1.0-2.9)$ \\
\hline \multicolumn{7}{|c|}{$\begin{array}{l}\text { 4. Fully adjusted for previous significant } \\
\text { factors and child's demographic factors } \\
\text { reported at } 21 \text {-year follow-upa,e }\end{array}$} \\
\hline Live birth & $2.1(1.2-3.8)$ & $0.9(0.5-1.7)$ & $0.8(0.4-1.5)$ & $1.0(0.4-2.6)$ & $0.7(0.4-1.2)$ & $1.0(0.6-1.6)$ \\
\hline Abortion & $2.5(1.5-4.2)$ & $2.1(1.3-3.5)$ & $1.5(0.9-2.5)$ & $3.6(2.0-6.7)$ & $1.5(0.9-2.4)$ & $1.5(1.0-2.4)$ \\
\hline Miscarriage & $2.1(1.2-3.9)$ & $1.7(0.9-3.1)$ & $1.4(0.8-2.5)$ & $2.6(1.2-5.4)$ & $1.4(0.8-2.4)$ & $1.5(0.9-2.5)$ \\
\hline \multicolumn{7}{|c|}{$\begin{array}{l}\text { a. Reference group - never pregnant; obtained using binominal logistic regression. } \\
\text { b. Adjusted for early life and family factors (birth father's presence at 14, mother-child communication at 14, birth mothers education, income level, age at the time of first live birth, } \\
\text { and history of chronic depression, anxiety (Delusions-Symptoms-States Inventory) and smoking during pregnancy). } \\
\text { c. Adjusted for adolescent behaviours reported at the 14-year follow-up: aggression/delinquent behaviour (Child Behavior Checklist), anxious/depressed behaviour (Youth Self- } \\
\text { Report), child-reported performance at school, cigarette smoking and alcohol use. } \\
\text { d. Adjusted for factors reported at the } 21 \text {-year follow-up: age of first sexual intercourse, sexual abuse before } 16 \text { years of age, reported ever being raped, and age when cannabis } \\
\text { was first used (cannabis use excluded in cannabis DSM-IV disorder). } \\
\text { e. Adjusted for demographic factors at 21: age, education level, living arrangements, marital status and the age the young adults first left the family home. }\end{array}$} \\
\hline
\end{tabular}

removed. Exclusion of participants reporting cigarette and alcohol use, anxious/depressed behaviour and aggressive/delinquent behaviour at 14 yielded similar results to those presented in Table 2.

As timing of the pregnancy was not recorded, young women reporting onset of a disorder at or before the age of their debut sexual intercourse were excluded. The majority of young women had onset of DSM-IV depression $(55.8 \%, n=192)$ and anxiety $(87.6 \%, n=366)$ before they started having sexual intercourse. Removal of those with early onset strengthened associations between abortion and depression $(\mathrm{OR}=1.9,95 \% \mathrm{CI} 1.1-3.3)$ and all the pregnancy outcomes and anxiety $(\mathrm{OR}=2.9,95 \%$ CI $1.2-$ 6.7). The remaining results were essentially unchanged from those reported in Table 2 (online Table DS4).

A sensitivity analysis using current (12-month) DSM-IV disorders was conducted to better account for the temporal sequence of pregnancy outcomes, psychiatric and substance use disorders. The findings were not substantially different from Table 2 , except the association between abortion and current affective disorder became significant ( $\mathrm{OR}=1.9,95 \%$ CI 1.1-3.2) (online Table DS5).

\section{Attrition}

There was significant loss to follow-up between the 14- and 21year surveys. To determine if loss to follow-up at 21 years affected the validity of our findings, we conducted a sensitivity analysis using inverse probability weights ${ }^{20}$ modelling the probability of having missing data at age 21 . Relevant variables available at baseline were included in an exploratory regression model to determine whether individuals remaining in the study significantly differed from those lost to follow-up. Loss to follow-up at age 21 was predicted by being the child of a teenage mother
$(\mathrm{OR}=1.8,95 \%$ CI $1.5-2.0)$ who smoked $(\mathrm{OR}=1.4,95 \%$ CI $1.3-$ $1.7)$ and reported anxiety symptoms during pregnancy $(\mathrm{OR}=1.4$, 95\% CI 1.2-1.7) (online Table DS1). When weights using factors that predicted missing data at age 21 were included in all adjusted models, the results were virtually the same as those presented here, except the association between lifetime alcohol dependency and miscarriage became significant ( $\mathrm{OR}=1.9,95 \% \mathrm{CI} 1.1-3.5$ ) (online Table DS5).

\section{Discussion}

\section{Main findings}

This study found that young women with a lifetime history of either abortion or miscarriage were at increased risk of tobacco dependence and illicit drug use (excluding cannabis) compared with women who had never been pregnant. Women who gave birth were also at similar increased risk of tobacco dependence. However, it appears that pregnancy and parenting tasks may discourage young women from heavy drug and alcohol use. ${ }^{21}$ Women who had an abortion were twice as likely as women who were never pregnant or who gave birth to have an alcohol disorder and current affective disorder. Associations remained robust after adjustment for a range of confounders and a series of sensitivity analyses.

Two prospective longitudinal studies, one from New Zealand ${ }^{2}$ and the other from Norway, ${ }^{3}$ used standardised psychiatric interviews to examine a range of psychiatric and substance use disorders following abortion. Both found evidence of a causal association between abortion and later psychiatric and substance use disorders after controlling for a wide range of pre-pregnancy factors including pre-existing mental health. The New Zealand birth cohort study followed 506 women and found those 
reporting an abortion before age 21 had significantly higher rates of depression, anxiety, alcohol and illicit drug use by age 25 compared with women who had never been pregnant or women who had given birth. ${ }^{2}$ The Norwegian study followed 769 young women from age 15 to 27 and found abortion before age 20 was associated with later nicotine dependence, alcohol, cannabis and other illicit drug use in women having no stable relationship with the biological father. ${ }^{3}$ Our study found that women having an abortion had an increased risk of both lifetime and current DSM-IV diagnoses of alcohol, illicit substance use and current affective disorders but women giving birth had no such increased risk, except for tobacco dependency. Contrary to Pedersen, ${ }^{3}$ we found no association between abortion and cannabis disorder.

Despite this, our findings challenge the existing evidence of a causal link between nicotine, ${ }^{3}$ other illicit drug use, alcohol use disorders and abortion. ${ }^{2}$ Pairwise comparisons found that pregnancy outcomes were all equally associated with tobacco dependence; abortion and miscarriage exhibited similar associations with lifetime and current other illicit drug and lifetime alcohol use disorders. To date, no population-based study has compared adverse risk of psychiatric and substance use disorders in women reporting miscarriage and induced abortion. This study is the first large longitudinal cohort that has found a similar pattern of increased risk of alcohol and other illicit drug use disorders in women reporting either abortion or miscarriage compared with women who were never pregnant or had given birth. The findings suggest that the poor outcomes reported for women who had an induced abortion may be associated with pregnancy loss rather than simply the experience of abortion. Induced abortion and miscarriage are both stressful life events that have been shown to lead to anxiety, sadness and grief ${ }^{5,22}$ and, for some women, serious depression and substance use disorders. ${ }^{22-24}$

Although we were not able to fully explain the mechanisms behind these associations, a number of possible pathways were explored in sensitivity analyses. Early heavy alcohol, illicit drug use and depression have been linked to risky sexual activity, unwanted pregnancies and pregnancy complications, ${ }^{24,25}$ and could be on the causal pathway leading to both pregnancy loss and adverse psychiatric outcomes. After taking these factors into account and removing early onset disorders our findings remained robust. This suggests that our results were unlikely to be explained by reverse causality. Pregnancy loss and substance misuse may have shared risk factors. Unplanned pregnancy commonly co-occurs with individual and social risk factors such as early sexual activity, poor school performance, cigarette smoking, alcohol and illicit drug taking and behaviour problems. ${ }^{21,24,26}$ Although we may have not been able to control for all confounding, associations in our study remained robust after adjustment for a wide range of such potential confounding factors. Finally, pregnancy loss may be directly associated with an increased risk of later substance misuse, particularly if alcohol and illicit drugs are used to decrease emotional responses to the loss. ${ }^{2,3,27-29}$ Future longitudinal studies are needed to better understand the relationship of pregnancy loss with adverse psychiatric outcomes in young women.

\section{Limitations}

This study has a number of limitations. Contextual information related to circumstances of the pregnancy loss were not collected, so we could not account for associated factors such as reasons to abort, support received and gestational age at the time of the pregnancy loss. We had no access to medical records to investigate whether specific circumstances surrounding the termination, the timing of termination or other pregnancy-related events would confound associations between pregnancy loss and psychiatric disorders. Concern about possible underreporting of abortion has been raised. ${ }^{2,30}$ Data on abortion history was collected from a self-reported survey, and Jones \& Kost $^{31}$ found this method provides a more accurate abortion history than face-to-face interview. Women may have misclassified induced abortions as miscarriages; however, this appears unlikely as the reported rate of miscarriage was no higher than expected. ${ }^{32}$ It is also possible that the use of retrospective recall and concurrent assessment of pregnancy outcomes and DSM-IV disorders introduced some bias in our findings, although sensitivity analysis using more recent onset psychiatric disorders produced the same results as those shown in our main analysis. The high rate of attrition between the 14- and 21-year follow-up raised the possibility of bias in estimation of the magnitude of risk. Weighted analysis, which adjusted for factors associated with attrition at age 21, was not substantially different from the reported findings, which suggests that attrition bias is unlikely to have produced bias in our results. Finally, ours is a study of young women whose ages ranged from 18 to 23 years and therefore our results should not be generalised to older women or women of all ages.

Our study found that young women had similar increased risks of lifetime and current tobacco use, alcohol dependency and illicit substance use, although not cannabis, after an abortion or miscarriage compared with women who had never been pregnant. These associations may relate to common factors associated with pregnancy loss or early pregnancy, rather than being caused by the experience of induced abortion. Kaeleen Dingle, MPH, Rosa Alati, PhD, School of Population Health; Alexandra
Clavarino, PhD, School of Pharmacy; Jake M. Najman, PhD, School of Population
Health and School of Social Science; Gail M. Williams, PhD, School of Population Health, University of Queensland, Brisbane, Australia

Correspondence: Kaeleen Dingle, Level 2, Public Health Building, School of Population Health, University of Queensland, Herston Road, Herston, QLD 4006, Australia. Email: s4002827@student.uq.edu.au

First received 19 May 2008, final revision 8 Jul 2008, accepted 10 Sep 2008

\section{Acknowledgements}

The authors wish to thank the Mater-University of Queensland Study of Pregnancy (MUSP) principal investigators for access to the data, the MUSP21 data collection team, as well as all the mothers and child participants over the 21 years of the MUSP project.

The core study has been funded primarily by the National Health and Medical Research Council of Australia (NHMRC). NHMRC also funded A.C. (Capacity Building Grant), R.A. (NHMRC Career Development Award in Population Health, ID: 519721) and K.D. (NHMRC Public Health Postgraduate Research Scholarship, ID: 351654). The views expressed in the article are those of the authors and not necessarily those of any funding body.

\section{References}

1 Cougle JR, Reardon DC, Coleman PK. Depression associated with abortion and childbirth: a long-term analysis of the NLSY cohort. Med Sci Monit 2003; 9: $105-12$.

2 Fergusson DM, Horwood LJ, Ridder EM. Abortion in young women and subsequent mental health. J Child Psychol Psychiatry 2006; 47: 16-24.

3 Pedersen W. Childbirth, abortion and subsequent substance use in young women: a population-based longitudinal study. Addiction 2007; 102: 1971-8.

4 Gilchrist AC, Hannaford PC, Frank P, Kay CR. Termination of pregnancy and psychiatric morbidity. Br J Psychiatry 1995; 167: 243-8.

5 Major B, Cozzarelli C, Cooper ML, Zubek J, Richards C, Wilhite M, Gramzow RH. Psychological responses of women after first-trimester abortion. Arch Gen Psychiatry 2000; 57: 777-84.

6 Rees DI, Sabia JJ. The relationship between abortion and depression: new evidence from the fragile families and child wellbeing study. Med Sci Monit 2007; 13: CR431-6.

7 Schmiege S, Russo NF. Depression and unwanted first pregnancy: longitudinal cohort study. BMJ 2005; 331: 1303-7. 
8 Neugebauer R. Depressive symptoms at two months after miscarriage: interpreting study findings from an epidemiological versus clinical perspective. Depress Anxiety 2003; 17: 152-61.

9 Brockington I. Postpartum psychiatric disorders. Lancet 2004; 363: 303-10.

10 American Psychiatric Association. Diagnostic and Statistical Manual of Mental Disorders (4th edn) (DSM-IV). APA, 1994.

11 Najman JM, Bor W, O'Callaghan M, Williams GM, Aird R, Shuttlewood G. Cohort profile: the Mater-University of Queensland study of pregnancy (MUSP). Int J Epidemiol 2005; 34: 992-7.

12 World Health Organization. Composite International Diagnostic Interview (CIDI) Version 2.1. WHO, 1992.

13 Andrews G, Peters L. The psychometric properties of the Composite International Diagnostic Interview. Soc Psychiatry Psychiatr Epidemiol 1998, 33: $80-8$.

14 Bedford A, Foulds G. Delusions-Symptoms-States Inventory: State of Anxiety and Depression. nferNelson, 1978.

15 Spence SH, Najman JM, Bor W, O'Callaghan MJ, Williams GM. Materna anxiety and depression, poverty and marital relationship factors during early childhood as predictors of anxiety and depressive symptoms in adolescence. J Child Psychol Psychiatry 2002; 43: 457-69.

16 Barnes $\mathrm{H}$, Olson DH. Parent-adolescent communication and the circumplex model. Child Dev 1985; 56: 438-47.

17 Achenbach T. Manual for the Young Adult Self-Report and Young Adult Behavior Checklist. University of Vermont, Department of Psychiatry, 1997.

18 Achenbach TM, Howell CT, McConaughy SH, Stanger CP. Six-year predictors of problems in a national sample: III. Transitions to young adult syndromes. J Am Acad Child Adolesc Psychiatry 1995; 34: 658-69.

19 Sorenson SB, Stein JA, Siegel JM, Golding JM, Burnam MA. The prevalence of adult sexual assault: the Los Angeles epidemiologic catchment area project. Am J Epidemiol 1987; 126: 1154-64.

20 Hogan JW, Roy J, Korkontzelou C. Handling drop-out in longitudinal studies. Stat Med 2004; 23: 1455-97.
21 Jaffee S, Caspi A, Moffitt TE, Belsky J, Silva P. Why are children born to teen mothers at risk for adverse outcomes in young adulthood? Results from a 20-year longitudinal study. Dev Psychopathol 2001; 13: 377-97.

22 Klier CM, Geller PA, Ritsher JB. Affective disorders in the aftermath of miscarriage: a comprehensive review. Arch Wom Ment Health 2002; 5 : 129-49.

23 Gold KJ, Dalton VK, Schwenk TL, Hayward RA. What causes pregnancy loss? Preexisting mental illness as an independent risk factor. Gen Hosp Psychiatry 2007; 29: 207-13.

24 Martino SC, Collins RL, Ellickson PL, Klein DJ. Exploring the link between substance use and abortion: the roles of unconventionality and unplanned pregnancy. Perspect Sex Reprod Health 2006; 38: 66-75.

25 Ramrakha S, Caspi A, Dickson N, Moffitt TE, Paul C. Psychiatric disorders and risky sexual behaviour in young adulthood: cross sectional study in birth cohort. BMJ 2000; 321: 263-6.

26 Fergusson DM, Boden JM, Horwood L. Abortion among young women and subsequent life outcomes. Perspect Sex Reprod Health 2007; 39: 6-12.

27 Bradshaw $Z$, Slade $P$. The effects of induced abortion on emotional experiences and relationships: a critical review of the literature. Clin Psychol Rev 2003; 23: 929-58.

28 Broen A, Moum T, Bödtker AS, Ekeberg Ö. The course of mental health after miscarriage and induced abortion: a longitudinal, five-year follow-up study. BMC Med 2005; 3: 18-31.

29 Broen AN, Moum T, Bödtker AS, Ekeberg Ö. Predictors of anxiety and depression following pregnancy termination: a longitudinal five-year followup study. Acta Obstet Gynecol Scand 2006; 85: 317-23.

30 Reardon DC, Cougle JR. Depression and unintended pregnancy in the National Longitudinal Survey of Youth: a cohort study. BMJ 2002; 324: 151-2.

31 Jones RK, Kost K. Underreporting of induced and spontaneous abortion in the United States: an analysis of the 2002 national survey of family growth. Stud Fam Plann 2007; 38: 187-97.

32 de Vaus DA. Diversity and Change in Australian Families: Statistical Profiles. Australian Institute of Family Studies, 2004.

EXTRA 